\title{
Dynamics of longitudinal and transverse modes along the junction plane in GaAlAs stripe lasers
}

\author{
Mengel, F; Ostoich, V
}

Published in:

I E E E Journal of Quantum Electronics

Link to article, DOI:

10.1109/JQE.1977.1069332

Publication date:

1977

Document Version

Publisher's PDF, also known as Version of record

Link back to DTU Orbit

Citation $(A P A)$ :

Mengel, F., \& Ostoich, V. (1977). Dynamics of longitudinal and transverse modes along the junction plane in GaAlAs stripe lasers. I E E E Journal of Quantum Electronics, 13(5), 359-361.

https://doi.org/10.1109/JQE.1977.1069332

\section{General rights}

Copyright and moral rights for the publications made accessible in the public portal are retained by the authors and/or other copyright owners and it is a condition of accessing publications that users recognise and abide by the legal requirements associated with these rights.

- Users may download and print one copy of any publication from the public portal for the purpose of private study or research.

- You may not further distribute the material or use it for any profit-making activity or commercial gain

- You may freely distribute the URL identifying the publication in the public portal

If you believe that this document breaches copyright please contact us providing details, and we will remove access to the work immediately and investigate your claim 
[6] T. L. Paoli and P. A. Barnes, "Saturation of the junction voltage in stripe-geometry (AlGa)As double-heterostructure junction lasers," Appl. Phys. Lett., vol. 28, pp. 714-716, June 1976.

[7] W. B. Joyce, R. W. Dixon, and R. L. Hartman, "Statistical characterization of the lifetimes of continuously operated (AlGa)As double-heterostructure lasers," Appl. Phys. Lett., vol. 28, pp. 684-686, June 1976.

[8] B. C. DeLoach, Jr., B. W. Hakki, R. L. Hartman, and L. A. D'Asaro, "Degradation of cw double-heterostructure lasers at 300 K," Proc. IEEE, vol. 61, pp. 1042-1044, July 1973.

[9] T. L. Paoli, "Nonlinearities in the emission characteristics of stripe-geometry (AlGa)As double-heterostructure junction lasers," IEEE J. Quantum Electron., vol. QE-12, pp. 770-776, Dec: 1976.

[10] R. W. Dixon, F. R. Nash, R. L. Hartman, and R. T. Hepplewhite, "Improved light-output linearity in stripe-geometry doubleheterostructure (Al,Ga)As lasers," Appl. Phys. Lett., vol. 29, pp. 372-374, Sept. 1976.

[11] T. L. Paoli and J. E. Ripper, "Direct modulation of semiconductor lasers," Proc. IEEE, vol. 58, pp. 1457-1465, Oct. 1970.

[12] See, for example, M. R. Spiegel, Statistics. New York: Schaum, 1961, pp. 201-216.

[13] T. L. Paoli, "Intrinsic fluctuations in the output intensity of double-heterostructure junction lasers operating continuously at $300^{\circ}$ K," Appl. Phys. Lett., vol. 24, pp. 187-190, Feb. 1974.

[14] D. E. McCumber, "Intensity fluctuations in the output of CW laser oscillators. I.," Phys. Rev., vol. 141, pp. 306-322, Jan. 1966.

[15] H. Haug, "Quantum-mechanical rate equations for semiconductor lasers," Phys. Rev., vol. 184, pp. 338-348, Aug. 1969.

[16] T. L. Paoli and J. E. Ripper, "Observation of intrinsic quantum fluctuations in semiconductor lasers," Phys. Rev., vol. 2A, pp. 2551-2555, Dec. 1970.

[17] - "Observation of intrinsic quantum fluctuations in semiconductor lasers (abstract)," IEEE J. Quantum Electron., vol. QE-6, p. 325 , June 1970 .

[18] Further calculations which also predict this frequency depression in externally modulated lasers have recently been reported by W. Harth, "Properties of injection lasers at large-signal modulation," Arch. Elektron. U., vol. 29, pp. 149-152, Apr. 1975.

[19] D. J. Morgan and M. J. Adams, "Quantum noise in semiconductor lasers," Phys. Status Solidi, vol. 11, pp. 243-253, 1972.

[20] D. A. Kleinman, "Maser rate equations and spiking," Bell Syst. Tech. J., vol. 43, pp. 1505-1532, July 1964.

[21] Further support for this conclusion has also been obtained with unaged window lasers of a design similar to that used in [8]. In those devices which also exhibited intensity pulsations, no regions of reduced spontaneous emission were found.

[22] S. -I. Gonda and S. Mukai, "Degradation and intensity fluctuations in CW AlGaAs double-heterostructure junction lasers," IEEE J. Quantum Electron., vol. QE-11, pp. 545-550, July 1975.

\section{Correspondence}

\section{Dynamics of Longitudinal and Transverse Modes Along the Junction Plane in GaAlAs Stripe Lasers}

\section{F. MENGEL AND V. OSTOICH}

\begin{abstract}
Observations of the transient excitation of higher order transverse modes along the junction plane in DH GaAlAs stripe lasers during subnanosecond pulse modulation is reported. These modes are strongly excited at the onset of the light pulse, they decay during 200 ps, and reappear after $400-600 \mathrm{ps}$. The width of the longitudinal mode spectrum decays from 40 to $10 \AA$ during $0.5-1 \mathrm{~ns}$.
\end{abstract}

\section{INTRODUCTION}

This correspondence reports on the experimental investigations of the transient behavior of the fundamental and higher order transverse modes along the junction plane in DH GaAlAs stripe lasers which operate stably in the fundamental modes under dc conditions. The measurements were made both in pulsed operation, allowing the use of high current levels, and during continuous pseudorandom bit sequence (PRBS) modulation at $200 \mathrm{Mbits} / \mathrm{s}$.

Recently, attention has been given to the influence of fast pulse modulation on the width of the longitudinal mode

Manuscript received June 10, 1976; revised January 31, 1977. This work was supported by the Danish Government Fund for Scientific and Industrial Research.

The authors are with the Electromagnetics Institute, Technical University of Denmark, Lyngby, Denmark. spectrum [1], [2]. Experiments [2] have indicated that the spectral width during a pulse mainly is given by the spectrum at the start of the pulse, suggesting that the bias current should be sufficiently above threshold for only a few longitudinal modes to exist. The dynamic properties of the higher order transverse modes have received little attention so far, although this topic may be important for the coupling efficiency and pulse dispersion of a slightly overmoded glass fiber attached to the laser [3].

\section{EXPERIMENTS}

The lasers used in these experiments were commercial $\mathrm{CW}$ double heterostructure stripe geometry GaAlAs lasers with threshold currents in the range $190-250 \mathrm{~mA}^{1}$ and with stripe widths close to $10 \mu \mathrm{m}$. The lasers were driven by an avalanche transistor pulse generator giving current pulses with a risetime of $200 \mathrm{ps}$ and a halfwidth of $6 \mathrm{~ns}$ and an amplitude determined by fixed attenuators. These were superimposed on the end of a $30 \mu \mathrm{s}$ bias pulse, where sufficient thermal stability is obtained. The time-resolved spectra were obtained using a $1 \mathrm{~m}$ spectrometer and an avalanche photodiode ${ }^{2}$ cou- $^{-}$ pled to a sampling oscilloscope. Part of the light was focused onto another avalanche photodiode placed in front of the spectrometer to monitor the total light output. The overall risetime of the detection system was estimated to be 220 ps.

Fig. 1 shows three spectra obtained at different times relative to the onset of the light pulse. The bias current was 112

\footnotetext{
${ }^{1}$ Laser Diode Laboratories LCW 10.

${ }^{2}$ Telefunken BPW 28.
} 


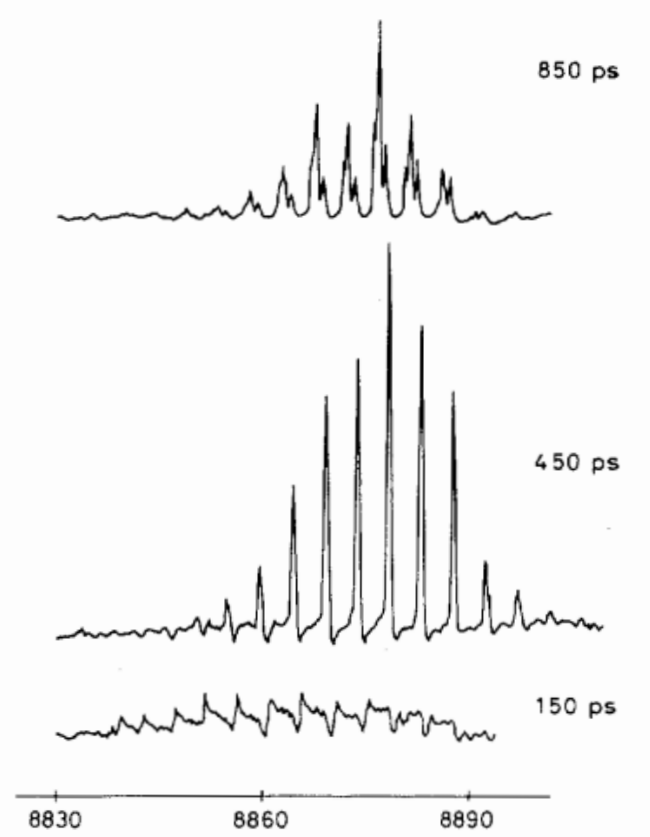

Fig. 1. Time-resolved spectra at different instants after the onset of a light pulse. Bias current $112 \mathrm{~mA}$, pulse amplitude $200 \mathrm{~mA}$, threshold current $112 \mathrm{~mA}$.

$\mathrm{mA}$, the pulse amplitude $200 \mathrm{~mA}$, and the threshold current $198 \mathrm{~mA}$. The usual relaxation oscillations of the light pulse are shown in Fig. 2(a), and the half power width of the longitudinal mode spectrum in Fig. 2(b). The width is seen to decay during approximately 1 ns to a stationary state corresponding to three longitudinal modes at a separation of 4.7 $\AA$. This behavior is only weakly dependent on the bias and pulse currents over a wide range, although the decay generally becomes faster for increasing bias currents. Fig. 2(c) shows the distribution of light power in the transverse modes as deduced from the time-resolved spectra. It is seen that highorder transverse modes are excited in the start of the pulse. They decay during a few hundred picoseconds, leaving the laser in the fundamental modes, but reappear after $600 \mathrm{ps,}$ dominating the laser emission completely. An unambiguous association of the spectrum with a particular transverse mode number is not possible in the start of the pulse, but in the spectrum at 850 ps the first- and second-order modes are clearly visible. The measured transition times between the regions of fundamental and higher order emission are limited by the response time of the detector. The transverse modes in the beginning of the pulse are generally excited more strongly the further below threshold the bias is chosen, but they appear even when biasing above threshold. The central wavelength of the envelope of the first-order modes is $10-5 \AA$ lower than that of the fundamental modes, the difference decreasing with increasing bias current. After 0.5-1 ns, the highorder modes reappear, dependent mainly on the total peak current but not on the relative value of the pulse and bias currents.

The laser from Figs. 1 and 2 was also modulated with a PRBS signal at $200 \mathrm{Mbits} / \mathrm{s}$ superimposed on a dc bias to check the validity of the results under more realistic drive conditions. Fig. 3 shows a typical pulse train in a 2048 bit sequence at a bias below (a) and above threshold (b) with constant modulated power. The spectrum during the last pulse in Fig. 3(a) is shown in Fig. 4, and the transverse modes can again be seen in the beginning of the pulse. These modes reappear at the end of the pulse. The spectrum during the first pulse in the sequence, which is preceded by a zero, was

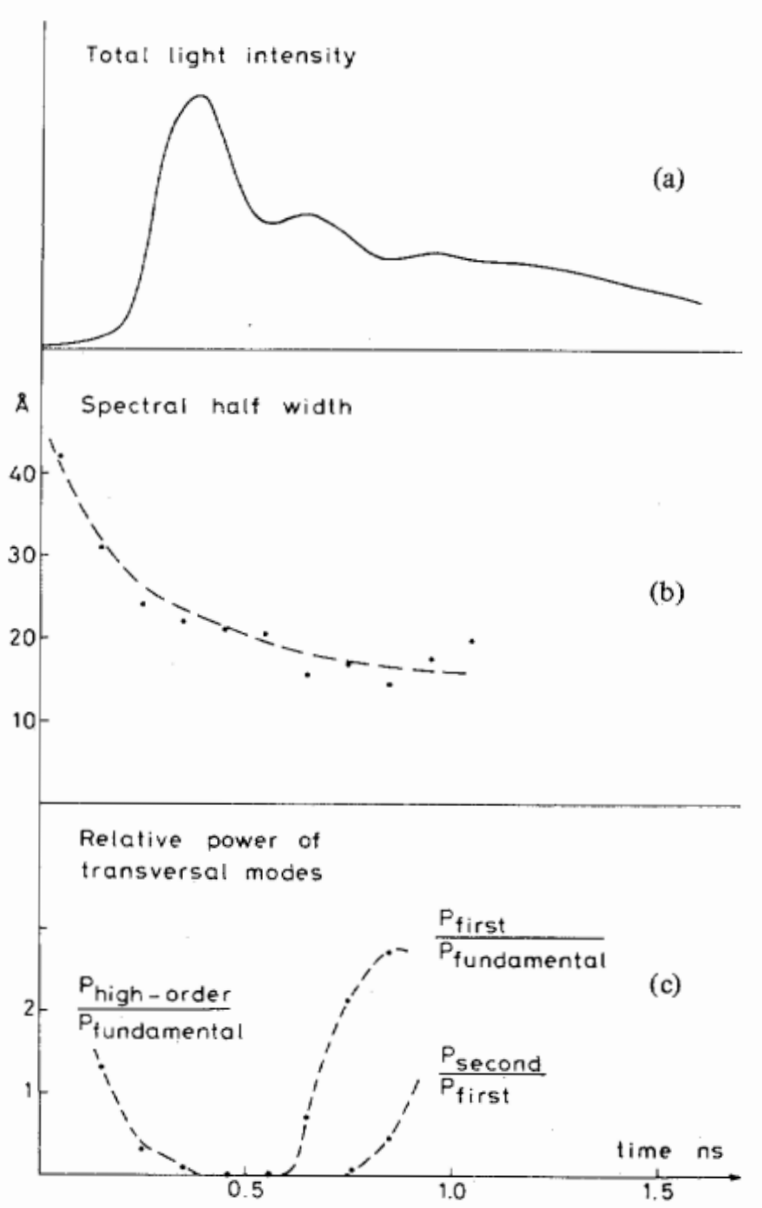

Fig. 2. Time evolution of laser emission in the pulse corresponding to Fig. 1. (a) Detected light pulse. (b) Half power width of the longitudinal mode spectrum. (c) Relative power in fundamental, firstand second-order transverse modes.

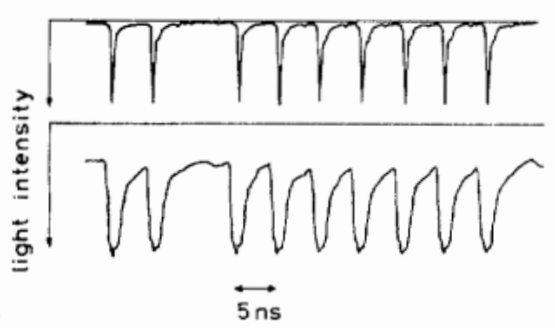

(a)

Fig. 3. PRBS modulation at $200 \mathrm{Mbits} / \mathrm{s}$. Threshold current $212 \mathrm{~mA}$, pulse amplitude $\approx 60 \mathrm{~mA}$. (a) Bias current $181 \mathrm{~mA}$. (b) Bias current $230 \mathrm{~mA}$.

similar to the one shown except for the fact that the transverse modes do not reappear due to the absence of the prepumping effect, visible in the last pulse as a "bump" on the trailing edge. When biasing above threshold in (b), the transverse modes are strongly excited during the pulse, even though the bias is not sufficient to reduce the spectral width below $30 \AA$. No significant dependence of the central wavelength of the envelope of the laser spectrum in a pulse on the preceding pulse pattern was found in the two cases in Fig. 3.

A possible explanation of these observations can be found by combining the usual rate equations [4] with the equations describing the carrier density profiles along the junction plane [5], [6]. When the bias current is far below threshold, the spontaneous emission will dominate and excite all modes al- 


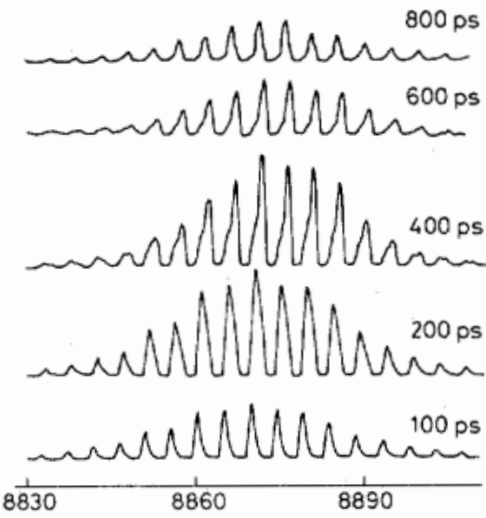

Fig. 4. Spectral evolution through the last pulse in Fig. 3(a) showing the presence of higher order transverse modes.

most equally. When a current pulse is applied, the electron density, and hence the gain in the part of the active layer located below the stripe contact, will grow, causing both fundamental and higher order transverse modes to be excited. Outdiffusion of carriers from the stripe edges will subsequently quench the first-order modes. The laser action in the fundamental modes will limit the electron density in the center of the stripe to a value close to the critical density [4], while the outer regions of the stripe will be pumped further, thus increasing the gain and hence the laser intensity in the firstorder modes. The absolute behavior depends on the magnitudes of the electron and hole diffusion constants as well as the photon lifetimes for the various transverse modes.

Computer simulations [7] based on this model have yielded results in qualitative agreement with the observations.

\section{CONCLUSION}

When a semiconductor stripe laser is modulated with fast pulses, it has been found that higher order transverse modes are excited in the start of each pulse. If the peak current is sufficiently high, these modes will reappear later in the pulses, eventually overtaking the laser emission. This phenomena may decrease the coupling efficiency to an optical fiber and cause fiber modes to be excited other than those present when the laser operates in the fundamental transverse modes. Due to the fact that as many as six longitudinal modes exist at currents up to 10 percent above threshold for the type of lasers tested, the spectrum during the pulses at $200 \mathrm{Mbit} / \mathrm{s}$ modulation had a width of $30 \AA$ even though the bias was chosen above threshold.

\section{REFERENCES}

[1]. T. Ikegami, "Spectrum broadening and tailing effect in directly modulated injection lasers," in Optical Fibre Communication, IEE Conf. Publ. 132, 1975, pp. 111-113.

[2] P. R. Selway and A. R. Goodwin, "Effect of dc bias level on the spectrum of GaAs lasers operated with short pulses," Electron. Lett., vol. 12, pp. 25-26, Jan. 8, 1976.

[3] G. D. Khoe, "Power coupling into single mode lasers," in Optical Fibre Communication, IEE Conf. Publ. 132, 1975, pp. 114-116.

[4] P. M. Boers, M. T. Vlaardingerbroek, and M. Danielsen, "Dynamic behavior of semiconductor lasers," Electron. Lett., vol. 11, pp. 206-208, May 15, 1975.

[5] B. W. Hakki, "Carrier and gain spatial profiles in GaAs stripe geometry lasers," J. Appl. Phys., vol. 44, pp. 5021-5028, Nov. 1973.

[6] - " "GaAs double heterostructure lasing behavior along the junction plane," J. Appl. Phys., vol. 46, pp. 292-302, Jan. 1975.

[7] J. Buus and M. Danielsen, "Carrier diffusion and higher order transverse modes in spectral dynamics of the semiconductor laser," submitted for publication.

\section{Surface Roughness and Laser Damage Threshold}

\author{
RICHARD A. HOUSE, II, JERRY R. BETTIS, AND
} ARTHUR H. GUENTHER

\begin{abstract}
The essential results of a carefully controlled experimental investigation of the effect of roughness on laser-induced surface damage thresholds in fused silica are reported. Sets of fused silica samples were subjected to several surface preparations such that rms surface roughness values within each set varied over two orders of magnitude. A plot of surface damage threshold versus surface roughness shows that there is a strong dependence of threshold on roughness, that this dependence exhibits a simple functional form, and that this relationship appears to hold even for the smoothest of optical surfaces. The utility of the observed threshold-roughness relationship is discussed.
\end{abstract}

Until recently, only a few experimental and theoretical observations have been made concerning the effects of surface roughness on laser-induced damage [1]-[4]. In 1975 [5], we presented the preliminary results of a series of carefully controlled experiments, using well-characterized samples, on the effects of surface topographic properties on laserinduced damage thresholds. We outline in this note the results of this surface roughness investigation.

Samples of fused silica were damage tested at $1.06 \mu \mathrm{m}$ using $\mathrm{TEM}_{00}$ pulses of 40.5 -ns pulsewidth produced by a laser system described elsewhere [5]. These samples were prepared as follows: conventional polish using barnesite (denoted as $\mathrm{CB}$ ); $\mathrm{CB}$ plus a short etch in dilute nitric acid; $\mathrm{CB}$ plus overcoat with a half-wave (at $1.06 \mu \mathrm{m}$ ) thickness of $\mathrm{MgF}_{2}$ or $\mathrm{SiO}_{2}$; and $\mathrm{CB}$ plus flame polish. Prior to polishing, all samples were control ground (see [5]) through a $3-\mu \mathrm{m}$ grit stage to minimize the subsurface disorder produced by grinding. Various final values of surface roughness were achieved by stopping the polishing operation after appropriate periods. Surface roughness values were measured by the techniques of fringes of equal chromatic order (FECO), total integrated scattering (TIS), and Talystep stylus measurements. Damage thresholds were determined by irradiating a virgin site each time the laser was fired; and all sites on each sample were shielded from each other against plasma blowoff and any resulting optical plasma radiation.

Fig. 1 presents a log-log graphical summary of the data, the vertical axis being the macroscopic breakdown electric field ${ }^{1}$ and the horizontal axis being the rms surface roughness. Bloembergen's $100-\AA$ limit [3] is identified to show that most of the present data fall below that value. It should be noted that surfaces with rms roughnesses considerably less than $100 \AA$ will usually still contain numerous surface asperities greater than the $100-\AA$ Bloembergen limit. Fradin's value for the laser-induced bulk damage threshold of fused silica [4] has been plotted by assuming the bulk "roughness" to be equal to the average interatomic spacing. Each data set has been bestfitted with a straight line, the equation of which is

$$
E \sigma^{m}=\text { constant. }
$$

Except for the flame polished samples, the value for $m$ centers around 0.5 for all the surface treatments. For the flame

Manuscript received January 5, 1977. This paper is based upon portions of a dissertation submitted in partial fulfillment of the requirements for the Ph.D. degree at the Air Force Institute of Technology, Wright-Patterson Air Force Base, OH (1975).

The authors are with the Air Force Weapons Laboratory, Kirtland Air Force Base, NM 87117.

${ }^{1}$ This electric field is the effective field at breakdown, produced in the surface of the material by the laser beam. It is termed macroscopic because it is the resultant, total electric field satisfying the boundary conditions (i.e., the Fresnel transmission relationship). 